\title{
A Wavelet Based Dissipation Method for ALE Schemes
}

Bill Cabot, Don Eliason, Leland Jameson

Lawrence Livermore National Laboratory

July 2000

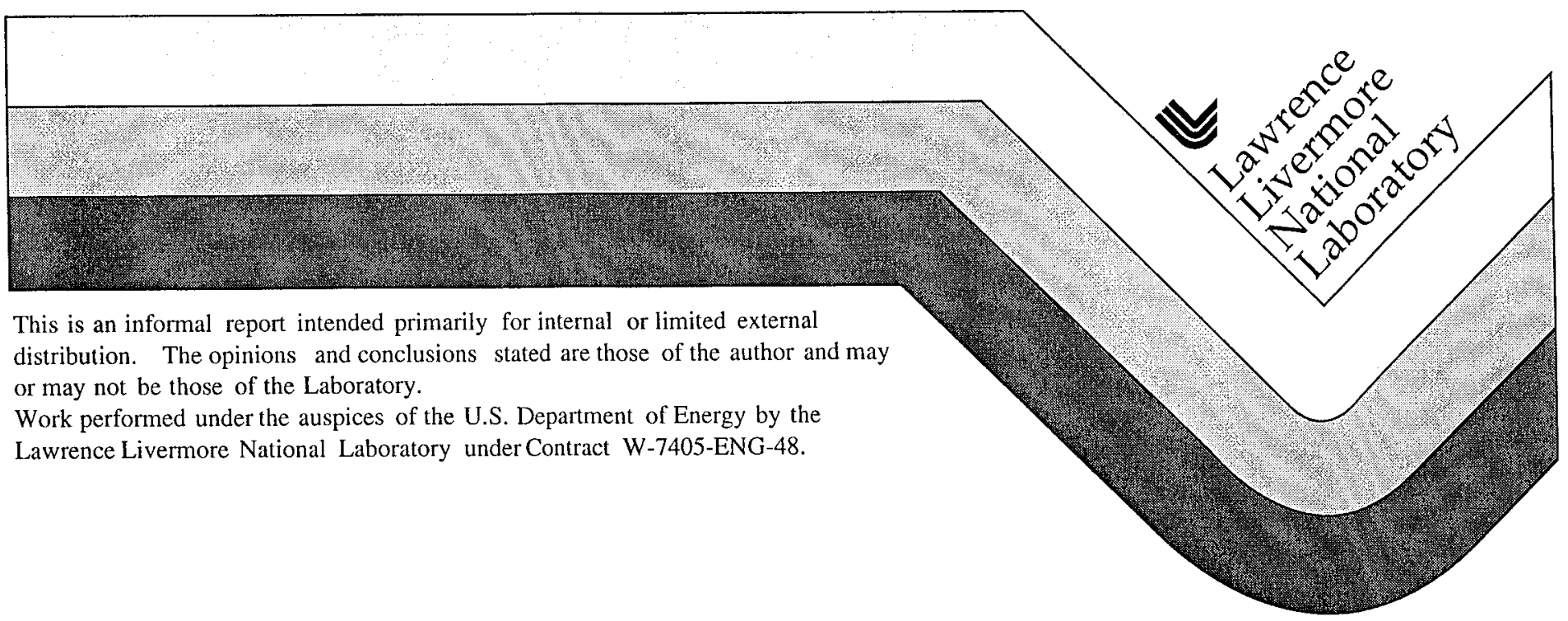




\section{DISCLAIMER}

This document was prepared as an account of work sponsored by an agency of the United States Government. Neither the United States Government nor the University of California nor any of their employees, makes any warranty, express or implied, or assumes any legal liability or responsibility for the accuracy, completeness, or usefulness of any information, apparatus, product, or process disclosed, or represents that its use would not infringe privately owned rights. Reference herein to any specific commercial product, process, or service by trade name, trademark, manufacturer, or otherwise, does not necessarily constitute or imply its endorsement, recommendation, or favoring by the United States Government or the University of California. The views and opinions of authors expressed herein do not necessarily state or reflect those of the United States Government or the University of California, and shall not be used for advertising or product endorsement purposes.

This work was performed under the auspices of the U.S. Department of Energy by the University of California Lawrence Livermore National Laboratory under contract No. W-7405-Eng-48. 


\title{
A Wavelet Based Dissipation Method for ALE Schemes
}

\author{
Bill Cabot \\ Don Eliason \\ Leland Jameson
}

\begin{abstract}
Wavelet analysis is natural tool to detect the presence of numerical noise, shocks and other features which might drive a calculation to become unstable. Here we suggest ways where wavelets can be used effectively to define a dissipation flag to replace dissipation flags traditionally used in ALE numerical schemes.
\end{abstract}




\section{Contents}

1 Introduction 3

2 Wavelets and Dissipation Flags 4

2.1 Defining the Daubechies Wavelet . . . . . . . . . . . . 4

2.2 Dissipation Flags in Wavelet Subspaces . . . . . . . . . . . 5

2.3 Filters, Orthogonality and Dissipation Flags . . . . . . . . 6

2.4 Implementation on a Computer . . . . . . . . . . . . 6

3 Dissipation Flags for Density and Pressure 8

4. Wavelet Information at Different Scales 11

5 Software Implementation 13

5.1 Generating the Wavelet Coefficients: lines 9-13 . . . . . . . . . 13

5.2 The Input and Output variables . . . . . . . . . . . . . . . 13

$\begin{array}{lll}6 & \text { Conclusion } & 18\end{array}$

\section{List of Figures}

1 Density (divided by 10), wavelet flag and divergence flag of $-\nabla \cdot u$ from the results of simulating the Sod, see $([6])$, shock using the Lagrangian code sKULL . . . . . . . . . . . . . . . 9

2 Pressure (divided by 10), wavelet flag and divergence flag. . . 10

3 Wavelet energy at different scales for a given pressure profile. 12 


\section{Introduction}

Numerical dissipation should be used sparingly and only in regions of a computational domain where it is numerically necessary. The definition of numerical necessity will be in terms of wavelet analysis. Wavelet analysis can give the practitioner a clear view of regions of the computational domain where numerical errors will be generated by small-scale oscillations or perhaps small scales in the regions of high gradients, see ([3]), ([4]), and ([5]). Sometimes dissipation flags are fundamentally built around criteria which are not numerical. These flags might work in some situations, but one can almost always construct an example where failure can be induced. Wavelet bașed dissipation flags, on the other hand, are constructed around the right fundamentals and are quite robust.

We first explore the reasons why wavelets work well for dissipation flags and then we show computational examples. 


\section{Wavelets and Dissipation Flags}

Possibly the most instructive way to think of wavelets is in contrast to traditional analysis techniques such as Fourier analysis. With Fourier analysis we analyze discrete or continuous data using basis functions which are global, smooth and periodic. This analysis yields a set of coefficients, say, $a_{k}$, which gives the amount of energy in the data at frequency $k$. Wavelet analysis, by contrast, analyzes data with basis functions which are local, slightly smooth, not periodic, and which vary with respect to scale and location. Wavelet analysis thereby produces a set of coefficients $b_{j, k}$ which give the amount of energy in the data at scale $j$ and location $k$. Wavelet analysis can serve as a good complement to Fourier analysis. In fact, data which is efficiently

analyzed with Fourier analysis often is not efficiently analyzed with wavelet analysis and the opposite situation also holds.

For our purposes here we will confine our discussion to the so-called orthogonal wavelets and specifically the Daubechies family of wavelets. The orthogonality property leads to a clear indication when data deviates from a low-order polynomial, the importance of which will become clear when we discuss numerical methods.

\subsection{Defining the Daubechies Wavelet}

To define Daubechies-based wavelets, see Daubechies 1988 and Erlebacher 1996 , consider the two functions $\phi(x)$, the scaling function, and $\psi(x)$, the wavelet. The scaling function is the solution of the dilation equation,

$$
\phi(x)=\sqrt{2} \sum_{k=0}^{L-1} h_{k} \phi(2 x-k),
$$

which carries the name "dilation equation" since the the independent variable $x$ appears alone on the left hand side but is multiplied by 2 , or dilated, on the right hand side. One also requires the the scaling function $\phi(x)$ be normalized: $\int_{-\infty}^{\infty} \phi(x) d x=1$. The wavelet $\psi(x)$ is defined in terms of the scaling function,

$$
\psi(x)=\sqrt{2} \sum_{k=0}^{L-1} g_{k} \phi(2 x-k) .
$$


One builds an orthonormal basis from $\phi(x)$ and $\psi(x)$ by dilating and translating to get the following functions:

$$
\phi_{k}^{j}(x)=2^{-\frac{j}{2}} \phi\left(2^{-j} x-k\right)
$$

and

$$
\psi_{k}^{j}(x)=2^{-\frac{j}{2}} \psi\left(2^{-j} x-k\right),
$$

where $j, k \in Z . j$ is the dilation parameter and $k$ is the translation parameter.

\subsection{Dissipation Flags in Wavelet Subspaces}

Generally speaking, proper numerical dissipation flags will come from the wavelet subspaces at the smallest scales. It is this energy at the smallest scales which can become unwanted oscillations or essentially information which is unresolved.

It is usual to let the spaces spanned by $\phi_{k}^{j}(x)$ and $\psi_{k}^{j}(x)$ over the parameter $k$, with $j$ fixed, be denoted by $V_{j}$ and $W_{j}$ respectively,

$$
\begin{aligned}
& V_{j}=\underset{k \in Z}{\operatorname{span}} \phi_{k}^{j}(x), \\
& W_{j}=\operatorname{span}_{k \in Z} \psi_{k}^{j}(x) .
\end{aligned}
$$

The spaces $V_{j}$ and $W_{j}$ are related by,

$$
\ldots \subset V_{1} \subset V_{0} \subset V_{-1} \subset \ldots
$$

and

$$
V_{j}=V_{j+1} \bigoplus W_{j+1},
$$

where the notation $V_{0}=V_{1} \oplus W_{1}$ indicates that the vectors in $V_{1}$ are orthogonal to the vectors in $W_{1}$ and the space $V_{0}$ is simply decomposed into these two component subspaces.

So, certainly the information in $W_{1}$ can lead directly to information which should be damped. But, one might also consider the information in $W_{2}$ and $W_{3}$ and the rate at which this information is headed toward $W_{1}$. 


\section{$2.3 \quad$ Filters, Orthogonality and Dissipation Flags}

The coefficients $H=\left\{h_{k}\right\}_{k=0}^{L-1}$ and $G=\left\{g_{k}\right\}_{k=0}^{L-1}$ are related by $g_{k}=(-1)^{k} h_{L-k}$ for $k=0, \ldots, L-1$. All wavelet properties are specified through the parameters $H$ and $G$. If one's data is defined on a continuous domain such as $f(x)$ where $x \in R$ is a real number then one uses $\phi_{k}^{j}(x)$ and $\psi_{k}^{j}(x)$ to perform the wavelet analysis. If, on the other hand, one's data is defined on a discrete domain such as $f(i)$ where $i \in Z$ is an integer then the data is analyzed, or filtered, with the coefficients $H$ and $G$. In either case, the scaling function $\phi(x)$ and its defining coefficients $H$ detect localized low frequency information, i.e., they are low-pass filters (LPF), and the wavelet $\psi(x)$ and its defining coefficients $G$ detect localized high frequency information, i.e., they are high-pass filters (HPF). Specifically, $H$ and $G$ are chosen so that dilations and translations of the wavelet, $\psi_{k}^{j}(x)$, form an orthonormal basis of $L^{2}(R)$ and so that $\psi(x)$ has $M$ vanishing moments which determines the accuracy. In other words, $\psi_{k}^{j}(x)$ will satisfy

$$
\delta_{k l} \delta_{j m}=\int_{-\infty}^{\infty} \psi_{k}^{j}(x) \psi_{l}^{m}(x) d x,
$$

where $\delta_{k l}$ is the Kronecker delta function, and the accuracy is specified by requiring that $\psi(x)=\psi_{0}^{0}(x)$ satisfy

$$
\int_{-\infty}^{\infty} \psi(x) x^{m} d x=0,
$$

for $m=0, \ldots, M-1$. Under the conditions of the previous two equations, for any function $f(x) \in L^{2}(R)$ there exists a set $\left\{d_{j k}\right\}$ such that

$$
f(x)=\sum_{j \in Z} \sum_{k \in Z} d_{k}^{j} \psi_{k}^{j}(x),
$$

where

$$
d_{k}^{j}=\int_{-\infty}^{\infty} f(x) \psi_{k}^{j}(x) d x .
$$

\subsection{Implementation on a Computer}

The wavelet decomposition matrix is the matrix embodiment of the dilation equation, Eq.(1), defining the scaling function and the accompanying equation defining the wavelet, Eq.(2). The following two recurrence relations for 
the coefficients, $s_{k}^{j}$ and $d_{k}^{j}$ are given as

$$
s_{k}^{j}=\sum_{n=1}^{L} h_{n} s_{n+2 k-2}^{j-1}
$$

and

$$
d_{k}^{j}=\sum_{n=1}^{L} g_{n} s_{n+2 k-2}^{j-1}
$$

as obtained from Eqs.(1)-(2), and we recall that $h_{n}$ refers to the chosen filter while we have $g_{n}=-(-1)^{n} h_{L-n}$. 


\section{Dissipation Flags for Density and Pressure}

Here we show two examples of the wavelet-based dissipation flag applied to the pressure and density fields. Note that wavelet analysis is fundamentally built around defining regions of the domain where a numerical calculation is on the verge of failing due to insufficient grid point density. No other regions will be flagged, only those that need dissipation. In Figure (1) we can clearly see that the wavelet flag identifies the regions where either grid points are needed or dissipation should be applied. Further, the magnitude of the flag is proportional to the amount of dissipation that is needed. In other words, very sharp corners need more dissipation than smooth corners. In a sense this is obvious, but wavelet analysis gives us a very precise and automatic way to apply the dissipation for any calculation and, in particular, ALE schemes. In the figures, one will also find the divergence of the field plotted. Sometimes, as in the Lagrangian code of Figures (1) and (2), the divergence is used as a flag for dissipation but the divergence, or negative divergence, is fundamentally the wrong quantity to flag. One might be attempt to $f i x$ a flag built around the divergence, but it makes more sense to flag on the correct quantity from the beginning. 


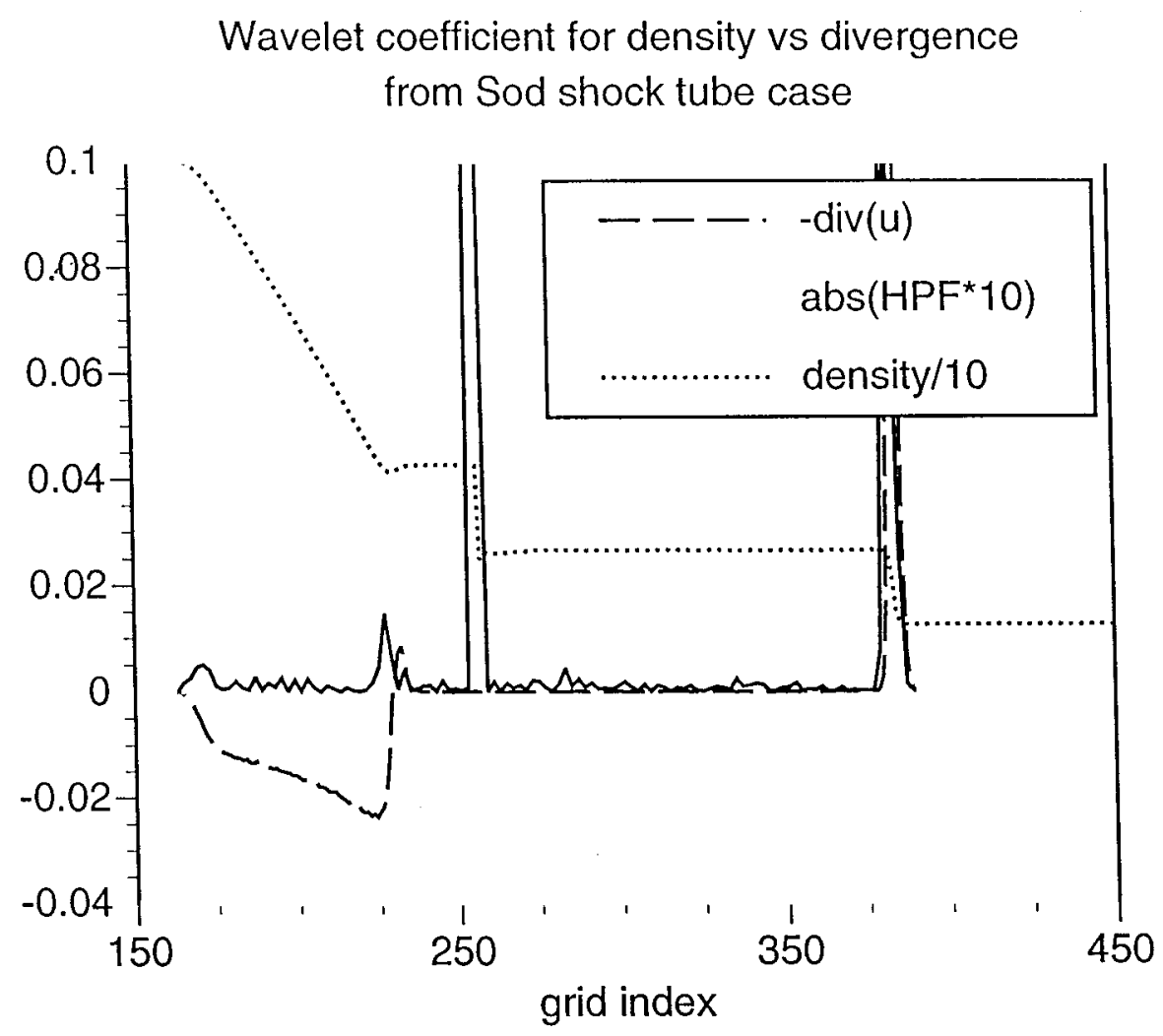

Figure 1: Density (divided by 10), wavelet flag and divergence flag of $-\nabla \cdot u$ from the results of simulating the Sod, see ([6]), shock using the Lagrangian code sKULL 


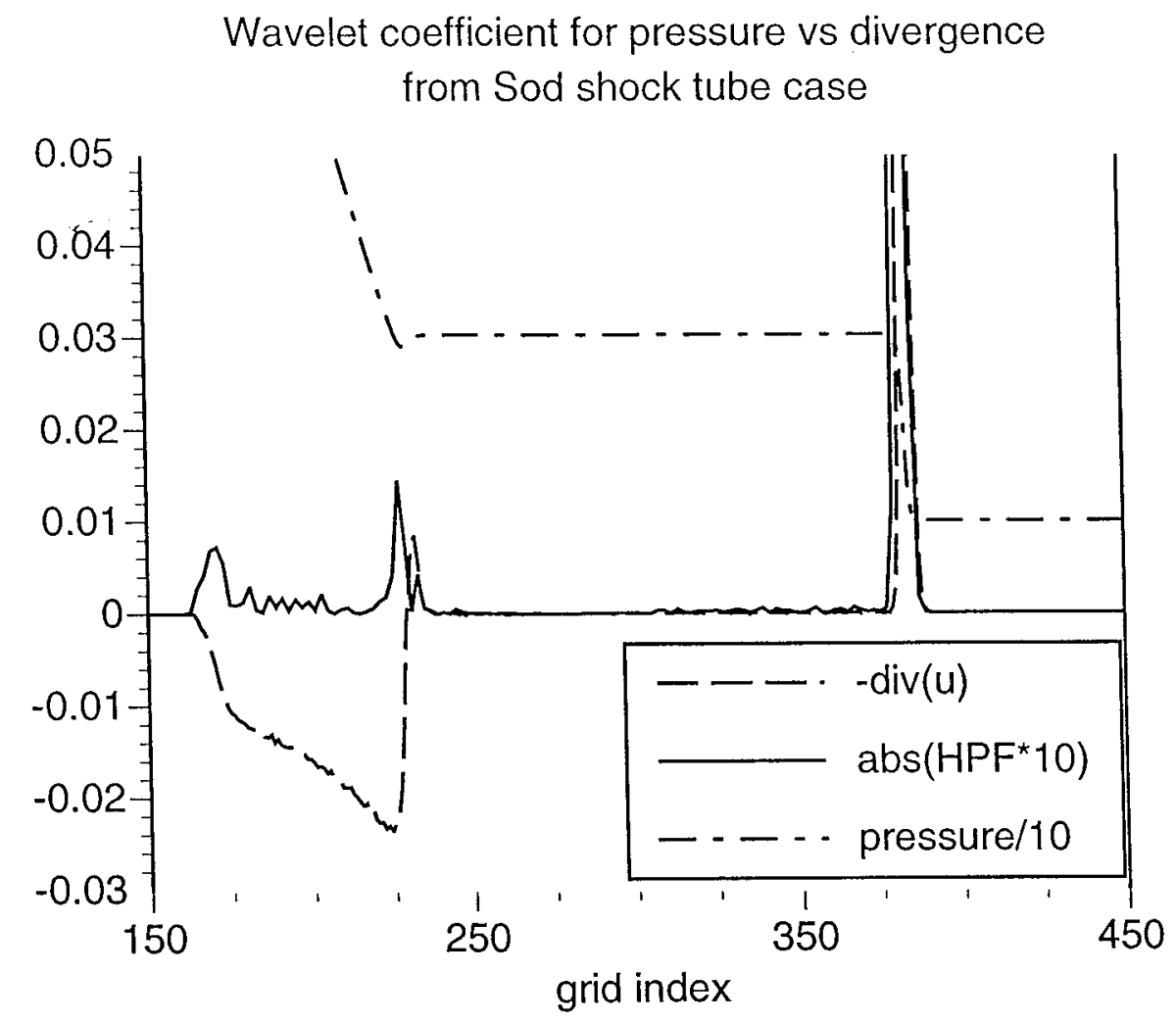

Figure 2: Pressure (divided by 10), wavelet flag and divergence flag. 


\section{Wavelet Information at Different Scales}

One of the key strengths of wavelet analysis is the ability to easily break up a signal into energy contributions at different scales at different locations in a domain. This ability gives the user an easy method to really understand what is happening in the computational domain. In a word, various structures have unique signatures in a wavelet basis. Such structures would be shocks, noise, smooth laminar flow, and essentially anything that can appear. Noise, for example, would have most of its wavelet energy in the finest scale with relatively little energy in the more coarse scales. A shock, by contrast, would have a great deal of energy at all scales but at only one location. In a sense, wayelets give the user all the information that one could hope for. In Figure (3) we can see these ideas illustrated for a given pressure profile. This profile has a shock, a region dominated by noise, and a non-shock but steep gradient region. The energy in the wavelet coefficients is plotted and one can see that these features are easily distinguished. This gives the user the knowledge needed to know where to damp and tells exactly where the shock it so that proper measures can be taken. 


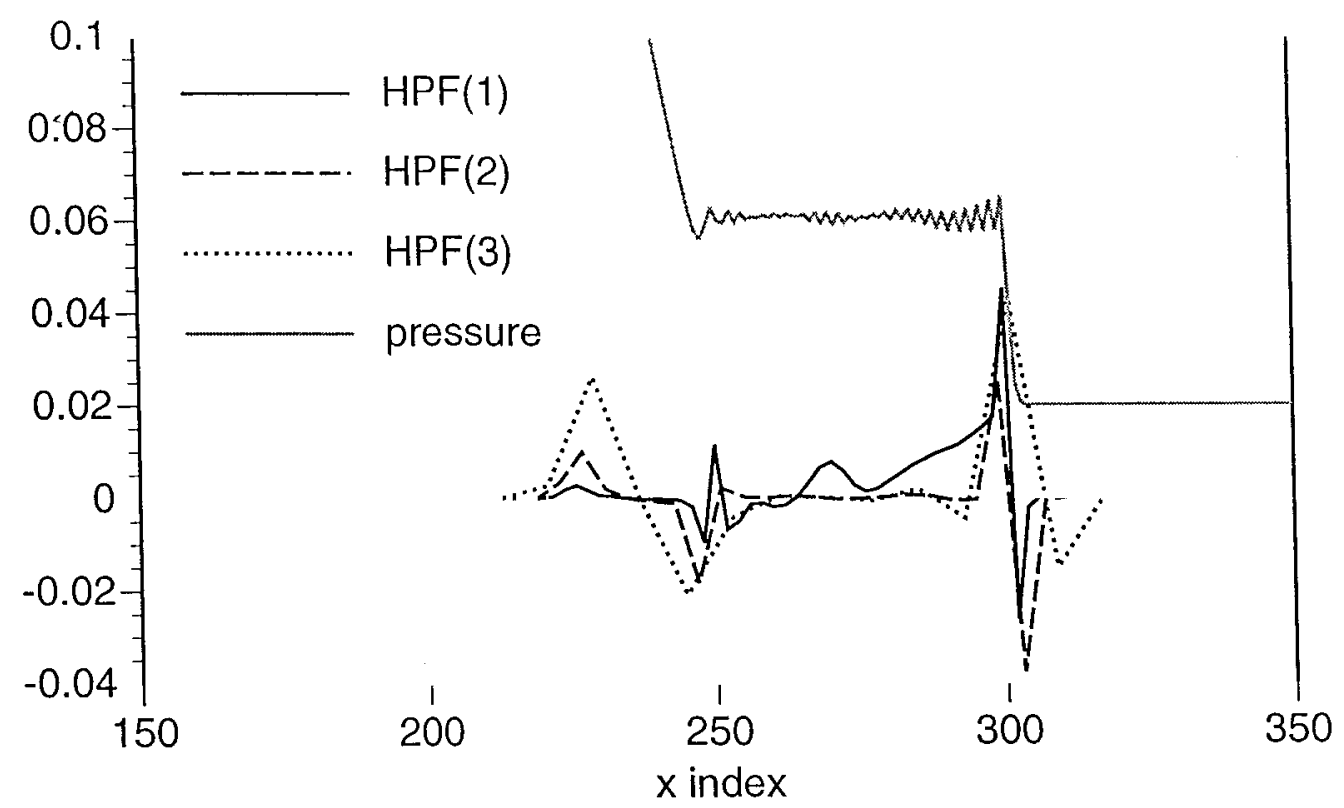

Figure 3: Wavelet energy at different scales for a given pressure profile. 


\section{Software Implementation}

This section gives a simple version of one possible implementation of the software that would be needed for the wavelet-based dissipation mechanism.

\subsection{Generating the Wavelet Coefficients: lines 9-13}

To illustrate, assume that the user has determined that 8 is the maximum desired ratio between the maximum $\Delta x$ and the minimum $\Delta x$. As noted above, this corresponds to 3 wavelet decompositions. As above, let $V_{0}$ denote the subspace spanned by scaling functions on the finest scale. One wavelet decomposition produces the division of $V_{0}$ into $V_{1}$ and $W_{1}: V_{0}=W_{1} \oplus V_{1}$. Similarly, three wavelet decompositions produces, $V_{0}=W_{1} \oplus W_{2} \oplus W_{3} \oplus V_{3}$. Each of these wavelet decompositions is performed by the subroutine filter.f. The input to filter. $f$ is the variable Extdata which contains the scaling function coefficients for subspace $V_{i}$ and the output variables of filter.f are the variables data and HPF which contain the coefficients for the subspaces $V_{i+1}$ and $W_{i+1}$, respectively. The variable is named Extdata because the coefficients of $V_{i}$ have been 'extended' to reflect the boundary conditions. In this version of the program, the data is extended by adding constant scaling function coefficient values to the ends of the vector data by the routine constext.f. Note, if one desires periodic boundary conditions then one 'wraps' the scaling function coefficients around such that one extends the vector data by returning to the beginning of the same vector. Likewise, if one wants a smoother extension of data then one can write a routine which extends linearly or by some other higher order polynomial.

\subsection{The Input and Output variables}

\section{Input Variables}

- $x i=$ The evenly-spaced grid point values.

- $\mathrm{fi}=$ The evenly-spaced samples of the function which is to be analyzed. $\mathrm{fi}(1)=$ value at left-hand boundary. $\mathrm{fi}(\mathrm{N}+1)=$ value at right-hand boundary. If boundary conditions are periodic, $f(1)=f(N+1)$.

- $\mathrm{L}=$ Defines which wavelet is used. For Daubechies $4, \mathrm{~L}=4$. 
- $\mathrm{N}=$ The number of points in $f i$ minus $1 . \mathrm{N}$ is a power of 2.

- $\mathrm{Nd}=$ Number of wavelet decompositions, e.g., if $\mathrm{Nd}=3$, then the ratio of the maximum $\Delta x$ to the minimum $\Delta x$ is $8=2^{3}$.

- $\mathrm{iw}=$ Width of wavelet refinement stencil. If $i w=1$, then the magnitude of wavelet coefficients are checked at three locations from -iw to iw or at the locations $-1,0,1$ in order to determine if the grid point at location 0 should be used. So that if one has a hyperbolic system, or traveling waves, then if $i w>2$ one can add grid points by looking 'backwards' and 'forwards' for a perturbation which might move into the region currently being examined. This is a kind of preparation for the future evolution of the system at hand.

\section{Output Variables}

- $\mathrm{HPF}=$ High Pass Filter and contains the results of the wavelet analysis. The first column will contain $\mathrm{N} / 2$ numbers indicating the result of wavelet analysis on the finest scale. The second column will contain the results from the analysis on the next coarsest scale and so on.

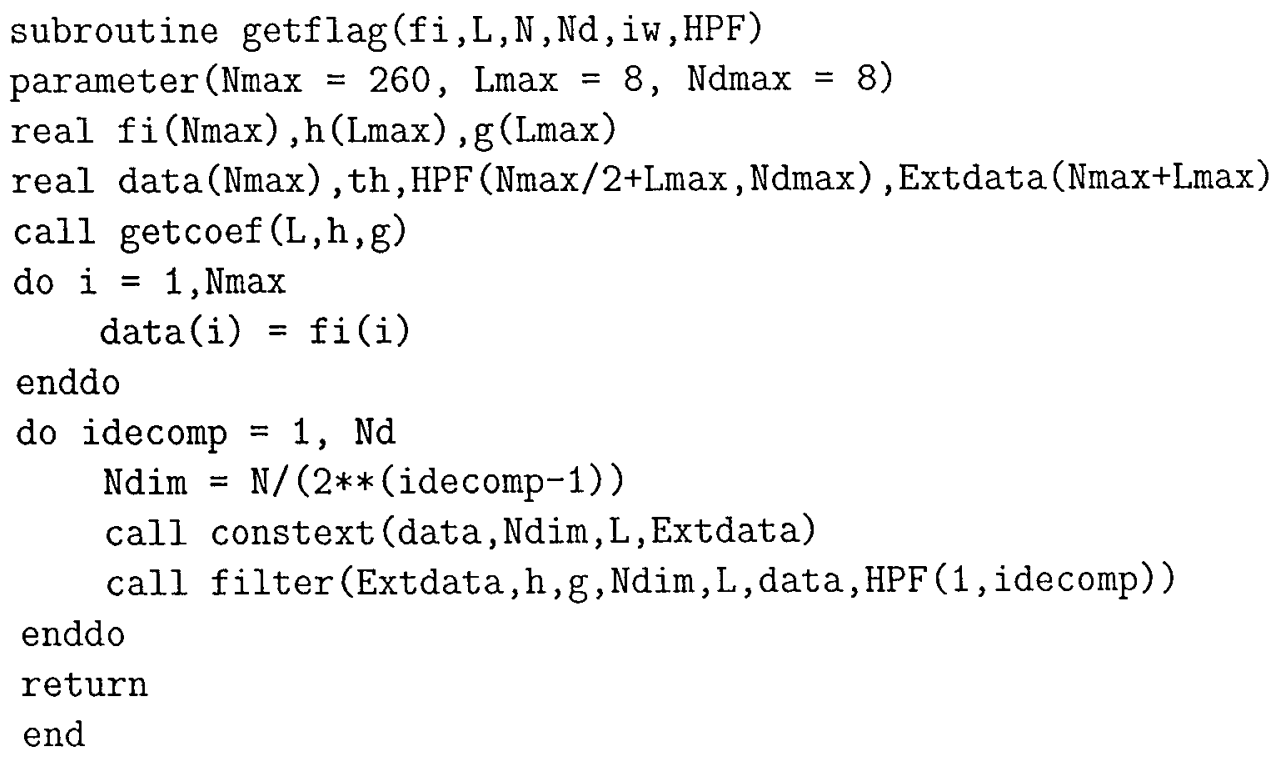




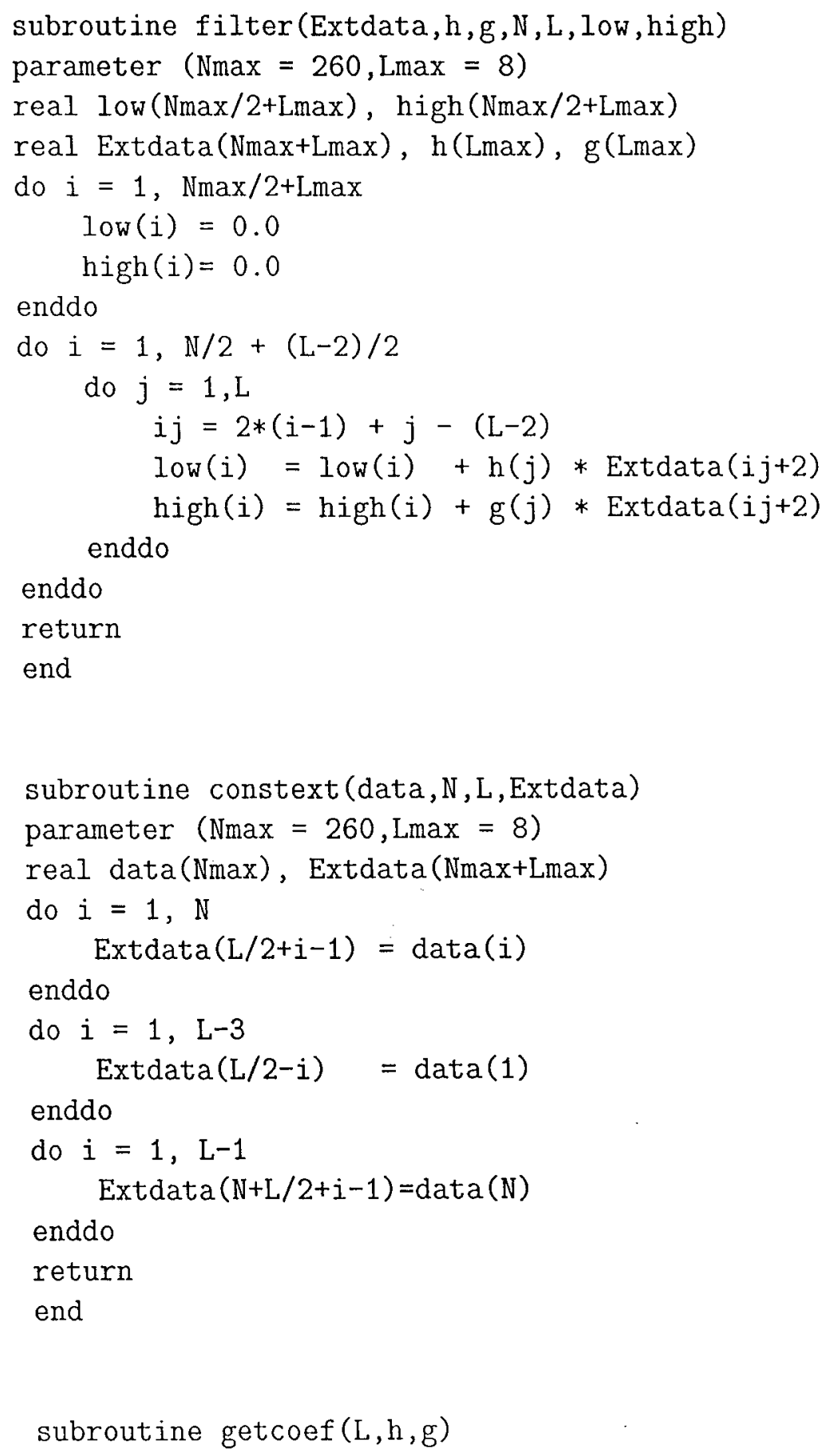




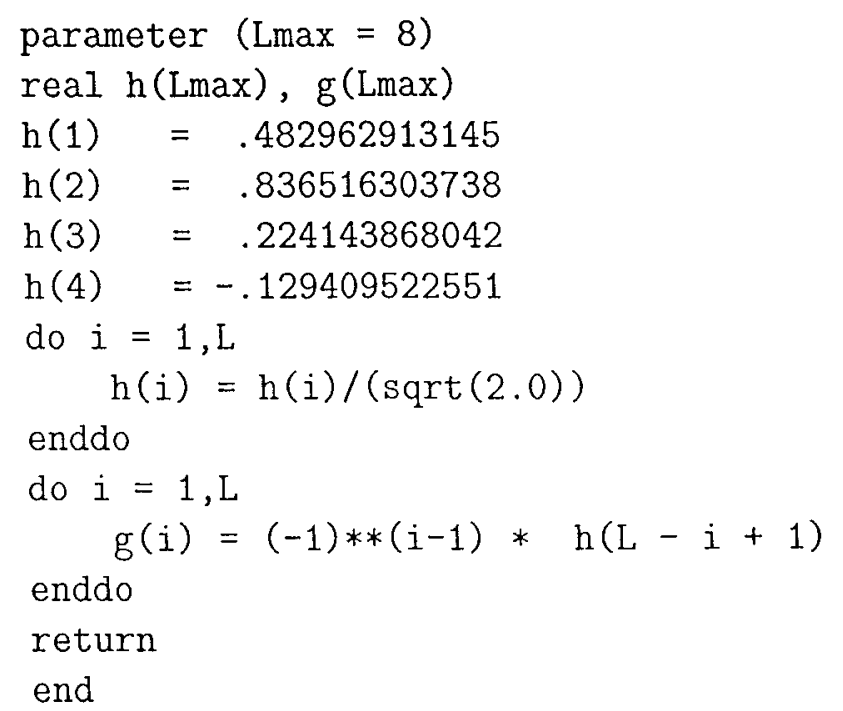




\section{References}

[1] I. Daubechies, (1988) "Orthonormal Basis of Compactly Supported Wavelets", Comm. Pure Appl. Math., 41 pp. 909-996.

[2] (1996) G. Erlebacher, M.Y. Hussaini, L. Jameson, "Wavelets: Theory and Applications", Oxford.

[3] L. Jameson, "Wavelets and Numerical Methods", PhD Thesis, Division of Applied Mathematics, Brown University, October, 1993.

[4] L. Jameson, "A Wavelet-Optimized, Very High Order Adaptive Grid . and Order Numerical Method", ICASE Report No. 96-30, and SIAM J. Sci. Comput., Vol. 19, No. 6, pp. 1980-2013, November 1998.

[5] L. Jameson, "On the Wavelet-Optimized Finite Difference Method", ICASE Report No. 94-9.

[6] G.A. Sod, "A Survey of Several Finite Difference Methods for Systems of Nonlinear Hyperbolic Conservation Laws", J. Comp. Phys. 27: 1-31. 


\section{Conclusion}

Numerical calculations sometimes require that numerical dissipation be added artificially in order to keep the calculation stable. It is critical, however, that this dissipation be applied sparingly and only where needed. The wavelet based method proposed here fulfills these requirements in a natural manner without requiring any ad hoc fixes for special cases. Wavelets detect exactly where a calculation will fail and tells the amount of dissipation that is needed to keep the calculation stable. The method is not only robust but computationally efficient. We have provided an basic introduction to the use of wavelet methods to enhance numerical calculations. Many of these ideas will be expanded on in later manuscripts. 